\title{
The Guidance of Scientific Experiment Record to Scientific Learning Method
}

\author{
Dang Fanwen a and Wang Qiang \\ Elementary Education College, Capital Normal University, Beijing, 100048,China \\ adangfanwen126@126.com, bqwchem@gmail.com
}

Keywords: Experimental record, Guidance, Learning method.

Abstract: The experimental record sheet is one of the essential elements of scientific experiments, which plays an important role in the process of scientific learning of primary school students. However, many schools and teachers ignore the effect of the experimental record sheet, and do not make full use of the records. This paper discusses that the experimental records can reflect the control variable method, analogy method, the method of seeking common induction, the method of covariant induction and other scientific learning methods, and these methods are very instructive for students to study science.

\section{Introduction}

In the process of scientific experiment teaching in primary school, each link has its necessity and importance. The experimental record sheet also plays a decisive role. The experimental task was designed to promote spontaneous record keeping compared to previous task designs by increasing the saliency of task requirements, with the design goal of making the relationship between record keeping and inquiry strategies more explicit and visible[1]. The experimental record can reflect the thinking process of students' experiment inquiry, and it is also an important carrier to cultivate students' literacy. The ability to collect evidence and process information in the scientific research objectives presented in the "Full-time Compulsory Education Primary Science Curriculum Standards" is actually the ability of the students to use the experimental records. Students are required to understand the methods of thinking, such as synthesis, comparison, classification, generalization, reasoning, analogy and so on, which can be well documented in the experimental record[2]. However, in practical teaching, many teachers ignore the role of experimental records, and students also have a negative and burnout psychology of the experimental records. The reason for this phenomenon is that science teachers did not find the importance of the experimental record sheet to the guidance for students' learning methods, and they have no awareness of the record's influence on the cultivation of students' Scientific Literacy.

\section{The status and function of the experimental records in primary school science experiments}

Observations and experiments are the two main ways to learn science for the students in primary school, and the record of experimental records is a true reflection of scientific experiments. Whether a scientist or a pupil, when they do scientific experiments, experimental records must be done. The record of scientific experiment is not only a diary of the purpose, the plan, the whole research 
process and the research results, but also the reflection of the objective facts and the presentation of scientific facts. For scientists, the experimental record is the crystallization of their hard work and wisdom; For students, the scientific record is the experimental "evidence" of each experiment that they have done. The design of the experimental record is a real reflection of the experimental design. Teachers can discover the progress and problems of the students through the experimental record. They can also penetrate learning methods and ways of thinking through the design of the experimental record to students and guide them. Students describe the experiment objectively through experiments records, and use experience to process the collected data, then they can get a reasonable explanation for objects change, the relation and development law between things to draw a scientific conclusion.

The experimental record can make the scientific inquiry process more profound. The method of scientific inquiry learning is one of the most popular scientific teaching methods that is highly praised by many teachers. When entering the inquiry-based teaching,the students pay attention to the problems of nature, collect data through their own research activities, and use the obtained information under the help of teachers to answer the questions.In this process, students not only construct scientific knowledge, but also learn how to study and solve problems[3]. There are usually two forms to design experimental record: one is the scientific experimental record of teachers was designed well in advance and provided directly to the students in the experimental class; The other one is that according to the guidance of teachers, students design their own experiments. Which way to chose depends on the specific content of courses and students. This paper mainly discusses the guiding role of the teachers' design record sheet to the students' learning methods.

\section{The teachers guide students learning methods through the experimental record}

\subsection{The control variable method in the experimental record sheet}

The control variable method is turning a multi-factor problem into a number of single-factor problems. Each time only one of the factors needs to be changed, the rest of the factors remain unchanged. And then the impact of this factor on things that have been changed will be studied, at last the impact of each changed factor will be studied integrally and problems can be solved finally. This method is called the control variable method.It is an important way of thinking in scientific research, and widely used in a variety of scientific exploration and scientific research. In the primary school, science teachers do not explain the control variable method, but students can experience it in the experimental process, the experimental record is one of the teachers' carrier to infiltrate this method.

For example, when teachers teach the "pendulum of the study" in the fifth-grade published by Educational Science Publishing House, they need to use a single pendulum to do the experiment.When a teacher designs the experimental record, it is necessary to predict what students think about the speed of the pendulum swing. The first factor is "whether the weight of the pendulum affects the speed of the pendulum", and let the students know which is a variable (this is the problem that needs to be studied), which factors are the invariants that needs to be controlled.The irrelevant factors need to be controlled, thus the accuracy of the research results can be ensured. The main factor to be controlled here is the length of the pendulum and the size of the swing, the teacher should make students understand that controlling invariants is as much as possible to control the invariant in the experimental environment, such as the direction of the swing, the size of the pendulum. (choosing from the pendulum's shape, mass and volume, etc.)In addition the operator is not supposed to get too close to the pendulum when doing the experiment. It is better that the same person control the swing of pendulum. The second factor is "whether the length of the swing rope affects the speed of the swing," which is similar to study of the first problem. The 
length of the rope is the variable that needs to be changed, but other factors, such as pendulum quality and swing, are required to be controlled strictly.

The experimental record provided by the teacher is designed from the method of embodying the control variable. As shown in the following table, according to the table 1 and table 2 , the students can clearly see the variables and invariants, so as to understand the learning method used in this experiment. After completing the above two experiments, students discussed and analyzed both experiments, then the teacher used three progressive questions "Is the speed of the pendulum related to the length of the rope? Does the weight of the pendulum affect the speed of the pendulum? What kind of pendulum swing fast, what kind of pendulum swing slowly, how do we know?" In fact, students through the experiment is not difficult to draw the conclusion, and the key is to analyze the data obtained by the students, and it is not difficult to draw a conclusion for students on the basis of the experimental record and analysis. The conclusions include: the swing speed of the pendulum is independent of the weight of the pendulum, and is related to the length of the pendulum. In the same pendulum, the longer the pendulum is, the slower the swing, and the shorter the swing is, the faster the swing.

Table 1: The Influence of Pendulum Length on the Swing Speed

\begin{tabular}{|c|c|c|c|c|}
\hline \multirow{2}{*}{$\begin{array}{c}\text { The Length of Simple } \\
\text { Pendulum }\end{array}$} & \multicolumn{3}{|c|}{ Experimental data(s/t) } & \multirow{2}{*}{ Average Swing Times } \\
\cline { 2 - 5 } & The First Time & The Second Time & The Third Time & \\
\hline Short & & & & \\
\hline Long & & & & \\
\hline Conclusion & & & \\
\hline
\end{tabular}

Table 2: The Influence of Pendulum Weight on the Swing Speed

\begin{tabular}{|c|c|c|c|c|}
\hline \multirow{2}{*}{$\begin{array}{c}\text { The Weight of the } \\
\text { Pendulum }\end{array}$} & \multicolumn{3}{|c|}{ Experimental data(s/t) } & \multirow{2}{*}{ Average Swing Times } \\
\cline { 2 - 5 } & The First Time & The Second Time & The Third Time & \\
\hline light & & & & \\
\hline heavy & & & & \\
\hline Conclusion & & & \\
\hline
\end{tabular}

Control variable method is a kind of covariant induction method, which is often used to do scientific experiments in the course of Science. As for primary school students, it is suitable for them to internalize scientific learning methods by themselves rather than being taught directly. The experimental record sheet is the key carrier of the scientific learning method with the visible guidance. 


\subsection{Seeking common and inductive method in experimental records}

Actually, scientific research is the process of seeking the causal relationship between nature and the object of study, induction is one of the main ways to find causality[4]. The inductive method is also called inductive reasoning, which is getting the conclusion that all objects of one kind of thing have a specific property based on the given fact that some objects of this kind have this certain property. Induction is a reasoning process which is from the particular to the general and it belongs to plausible reasoning. What's more, because the scope of the conclusion drawn by induction is greater than the scope of the premise, induction is the range of probability inference(probabilistic reasoning). The method of seeking common ground is also called the method of agreement. It refers to the situation that there always exists a factor as well as a same phenomenon in different environments and this factor has a causal relationship with this phenomenon[4].

The method of seeking common ground, which belongs to the induction method, can be used in scientific experiments to obtain a scientific understanding of the specific concept. This method can also be used to find the key features of a class of objects(things). Teachers can use this method when they design the experimental courses and experimental records sheets. Take the lesson How is the Sound Produced for example, which is from the textbook for Grade four of Education and Science Version, it is a need to use the induction method to find out the reasons of sound production. In detail, teachers need to guide the students to discover the common features existed in many familiar objects when they produce the sound (particular)through observing them so as to draw a general conclusion -- vibration produces the sound. The teacher can design the experimental record as shown in Table 3,"pressing the drum surface(drum-head)", "curving steel ruler" and "stretching rubber band"can not produce any sound. However, the sound can be produced by striking or stirring these same objects.As shown in the table 3, there is a common phenomena that some sounds are produced existed in the second experimental operation of the first three phenomena and that of the fourth phenomena. Meanwhile, that sound producing process accompanies another phenomena that there is always a vibration. In order to explore these conclusions, students will think about the commonalities of the four vocal phenomena. The method of seeking common ground is used inadvertently in this process.Therefore, teachers should be clear about the learning method sinvolved in the experimental process before they design the experimental records; moreover, they should pass these methods through the record sheets. 
Table 3: The Producing of Sound

\begin{tabular}{|c|c|c|c|c|}
\hline \multirow{8}{*}{$\begin{array}{l}\text { Experimental } \\
\text { Phenomena }\end{array}$} & & Experimental operation & $\begin{array}{l}\text { Whether or not it } \\
\text { sounds }\end{array}$ & $\begin{array}{l}\text { The principle of } \\
\text { making a sound }\end{array}$ \\
\hline & \multirow[t]{2}{*}{ Phenomenon I } & 1.Pressing the drum surface & & \\
\hline & & 2.Hitting the drum surface & & \\
\hline & \multirow[t]{2}{*}{ Phenomenon II } & 1.Curving the steel ruler & & \\
\hline & & 2. Toggling the steel ruler & & \\
\hline & \multirow[t]{2}{*}{ Phenomenon III } & 1.Stretching the rubber band & & \\
\hline & & 2. Toggling the rubber band & & \\
\hline & Phenomenon IV & $\begin{array}{l}\text { Taping the tuning fork to touch the } \\
\text { calm water. }\end{array}$ & & \\
\hline $\begin{array}{l}\text { Experimental } \\
\text { Results }\end{array}$ & & & & \\
\hline
\end{tabular}

\subsection{The method of covariant induction in experimental records}

The method of concomitant variation means that one phenomenon changes when a factor of the environment changes, and thus it is judged that this variable factor should be the reason why this phenomenon changes[4]. The covariant method is one of the ways to explore the causal relationship between phenomena. On some occasions where the phenomenon studied changes, if there is only one factor changes, this case is the cause of the phenomenon. The method of covariant induction is also one of the common scientific learning methods in primary school science experiments. This paper discusses the use of covariance method from the perspective of experimental record.

For instance, learning"the laws of light reflection" with providing some experimental equipment like laser pen, protractor, papers and pens for drawing. teachers provide and other experimental equipment. The experimental environment remains the same, and the only change is the angle between the incident ray and the normal (incident angle). Through changing the incident angle, it is obvious that the reflection angle becomes larger as the angle of incidence is larger, and the reflection angle becomes smaller as the angle of incidence is smaller.In order to study further that the relationship of size between the angle of incidence and the angle of reflection, teachers can design a specific form in the experimental record to ask the students to measure the size of the incident angle and the size of corresponding reflection angle and then make the conclusion that the size of reflection angle is equal to that of the angle of incidence by collecting a series of data. According to the characteristics of the experimental, teachers can teach students the method of the covariant induction through the design of experiment records. And this potential approach is essential for science learning. 


\section{Conclusions}

The experimental record sheets can guide the students to acquire not only three kinds of scientific learning methods, that is, the controlling variable method, the induction that seeking the common ground and the covariant induction; but also another two kinds of methods, deduction and the induction that seeking the differences. The experimental record about "The Producing of Sound" also reflects the learning method of comparison; what's more, it reflects that the controlling variable method can be found in the experiment which use the method of covariant induction. Above all, various learning methods can be used in one experiment or an experimental record at the same time. Teachers can make use of the experimental records to develop students' ways of thinking and cultivate their scientific literacy potentially. In addition, teachers can guide students to master a variety of scientific learning methods through the rational design of experimental records. Finally, the students complete the corresponding reasoning process by themselves, acquire the methods about science learning and apply these obtained learning methods into their later study life.

\section{References}

[1] Christopher Andersen and Nubia E. Rojo. Elementary Students’ Laboratory Record Keeping During Scientific Inquiry[J]. International Journal of Science Education. Volume 33,2011-Issue7.

[2] People's Republic of China Ministry of Education. Full Time Compulsory Education Primary School Science Curriculum Standards [S].2017

[3] [US] Arthur A. Carlin, Joel E. Bass, Terri L. Kang Tante. Teaching as Science as Inquiry [M]. People's Education Press.2008: 7.

[4] Ye Po. The Basis and Method for the Design of Primary School Science Teaching Observation Experiment [J]. Course $\cdot$ Teaching Material $\cdot$ Teaching Method.2013 (11): 68-72.

[5] Wang Qiang. Primary School Science Experimental Teaching Theory [M]. Beijing: People's Education Press .2015.

[6] "Ordinary logic" writing group. General logic [M]. Shanghai: Shanghai People's Publishing House .2011: 295-306.

[7] Wang Qiang, Sun Mingming, Zheng Ping, Zhang Mingzhu. Scientific Experiment - Teaching, Research, Study and Method [M]. Beijing: Science Press, 2013.

[8] You Changde. Use of Scientific Experiments to Record a Single [J]. Science of The Public (Science Education) .2015 (05): 75.

[9] Li Qing. Primary School Science High Quality Experimental Record Single Design [J]. Teaching and Management .2016 (08): 54-55.

[10] Zhang Yuping. Improvement and Optimization of Experimental Records of Science Experiment in Primary School [J]. Journal of Henan Institute of Education (NATURAL SCIENCE EDITION) 2014 (02): 76-80.

[11]A. F. What is This Thing Called Science [M]. Chalmers Science Press. 1982.

[12] Kang Sheng, Liu Mingsheng, Wei Na, et al. Experimental Record is an Important Means to Cultivate Students' Logical Thinking Ability [J]. Pharmaceutical Education.2011,27 (1): 61-63.

[13]Junqing Zhai.Jennifer Ann Jocz.Aik-Ling Tan. 'Am I Like a Science?':Primary Children'sImages of Doing Science in School.International Journal of Science Education [J]. Internationnal Journal of Science Education.2014(04):553-576. [14] Tan Dazhi, Zhang Zhen, Liu Xuefei, Chen Yufei. Translation of Experimental Records [J]. Laboratory Science .2012,12 (6): 206-208

[15] Liu Dachun. Philosophy of Science [M]. Beijing: Renmin University Press, 2011. 